\title{
Comments on the paper by Zinoviev and Bies "On acoustic radiation by a rigid object in a fluid flow"
}

\author{
F. Farassat, NASA Langley Research Center, Hampton, Virginia, U.S.A.
}

In a recent paper by Zinoviev and Bies in this Journal [1], the authors have claimed that the well-known theoretical results of Curle [2] and Ffowcs Williams and Hawkings (FW-H) [3] are incorrect. This claim is categorically refuted below and serious errors in [1] are pointed out.

\subsection{The Main Result of The Paper, its Origin and Relation to the Curle and the Ffowcs Williams-Hawkings Equation}

The authors start with a Kirchhoff-like formula for the density perturbation $\rho^{\prime}(\boldsymbol{x}, t)$, eq. (4), which they present without derivation or a reference. From the discussion in Subsection 3.1, one concludes that the authors intend to solve the radiation problem in the frame fixed to the solid surface $S$. Because of the fact that no Doppler factors are present in the denominators of the integrands of this equation, we conclude that the authors assume that the surface $S$ is stationary with respect to the undisturbed medium. Since the authors' two examples are for stationary solid objects, this assumption seems appropriate. The authors then manipulate eq. (4) algebraically to get their main result which is eq. (34). We have some objection about the fact that the authors have not clearly indicated the dependence of the integrands of eqs. (4), (34) and of all the other integrals on the retarded time. We will elaborate on this matter under another heading below.

Our first comment is on the origin of eq. (4). This equation is simply the sum of the solution of the Lighthill jet noise equation and the Kirchhoff formula for the density perturbations. The derivation of the Kirchhoff formula for the the acoustic density or pressure perturbations is based on the assumption that these variables satisfy the linear wave equation everywhere [4]. There is no mathematical or physical justifications from the first principles for the correctness of eq. (4) for the general flow situations where the Curle formula or the FW-H equation is valid. In fact when the correct procedures are followed, 
one is invariably led to the FW-H equation. From reference [5], slide 85, one can actually write down the inhomogeneous source terms of the wave equation for the acoustic density perturbation whose formal solution satisfies eq. (4) of the authors. For completeness we give this wave equation below:

$$
\square^{2} c_{0}^{2} \rho^{\prime}=\frac{\partial^{2} T_{i j}}{\partial x_{i} \partial x_{j}}-c_{0}^{2} \frac{\partial \rho^{\prime}}{\partial n^{\prime}} \delta(f)-\nabla \cdot\left[c_{0}^{2} \rho^{\prime} n^{\prime} \delta(f)\right]
$$

where $f(\boldsymbol{x})=0$ defines the surface $S$ with the assumption that $\nabla f=\boldsymbol{n}^{\prime}$ on this surface which can always be satisfied. Here $\boldsymbol{n}^{\prime}$ is the unit normal vector to $S$ pointing into the fluid. Note that the unit normal vector $\boldsymbol{n}$ in Zinoviev and Bies points into the surface $S$. We are using the established convention in the aeroacoustic literature for the direction of the unit normal to the surface $S$. The symbol $\delta(f)$ stands for the Dirac delta function with the support on the surface $S: f=0$. The uniform speed of sound in the undisturbed medium is denoted as $c_{0}$.

To carry the analysis further, we replace $c_{0}^{2} \rho^{\prime}$ everywhere in eq. (1) by the acoustic pressure $p^{\prime}$. The resulting equation is:

$$
\square^{2} p^{\prime}=\frac{\partial^{2} T_{i j}}{\partial x_{i} \partial x_{j}}-\frac{\partial p^{\prime}}{\partial n^{\prime}} \delta(f)-\nabla \cdot\left[p^{\prime} n^{\prime} \delta(f)\right]
$$

We now recognize two conditions depending on whether the surface $S: f=0$ is stationary or in motion with small amplitude.

1. The surface $S$ is rigid and stationary. In this case we have the boundary condition $\partial p^{\prime} / \partial n^{\prime}=0$. Then eq. (2) becomes:

$$
\square^{2} p^{\prime}=\frac{\partial^{2} T_{i j}}{\partial x_{i} \partial x_{j}}-\nabla \cdot\left[p^{\prime} \boldsymbol{n}^{\prime} \delta(f)\right]
$$

This equation is simply the linearized governing equation of the Curle formula. 
2. The surface $S$ is rigid and is in motion with small amplitude. Then the momentum equation gives

$$
-\frac{\partial p^{\prime}}{\partial n^{\prime}}=\rho_{0} \frac{\partial v_{n^{\prime}}}{\partial t}
$$

where $v_{n^{\prime}}$ is the local normal velocity of the surface $S$. Substituting this in eq. (2) gives the following equation:

$$
\square^{2} p^{\prime}=\frac{\partial^{2} T_{i j}}{\partial x_{i} \partial x_{j}}+\rho_{0} \frac{\partial v_{n^{\prime}}}{\partial t} \delta(f)-\nabla \cdot\left[p^{\prime} n^{\prime} \delta(f)\right]
$$

This is the linearized $F W$-H equation.

We see that in the limit of small perturbation, the eq. (4) of Zinoviev and Bies is equivalent to the linearized Curle formula when the surface $S$ is not in motion, and to the solution of the linearized FW-H equation when it is in motion with small amplitude. We note that these are really the well-known properties of the Kirchhoff formula in acoustics which is less general than the Curle formula and the solution of the FW-H equation.

\subsection{Analysis of The Two Examples in The Paper}

The two examples at the end of the paper are from linear acoustics. Zinoviev and Bies claim that even in these simple examples, the Curle's formula fails to give the correct results available in acoustics books. Furthermore, they claim their eq. (4) gives the correct results. The Curle formula should be applicable only to an example for which the surface $S$ is rigid and stationary. Example 1 of the paper satisfies this condition. However, as we demonstrate below, the authors have made some errors in manipulating the integrals of the Curle formula and erroneously blame the Curle formula for failing to give the correct result. We show below that Example 2 is in fact the determination of the radiation field of an oscillating sphere. Because in this example we have $\partial v_{n^{\prime}} / \partial n^{\prime} \neq 0$, the solution of the FW-H equation, or the linearized FW-H equation, should be used and not the Curle formula. It is not therefore surprising that the Curle formula should fail in this case although the authors again make some errors in manipulating the integrals of this formula. This 
discussion explains why the authors' eq. (4) works for both examples. The conclusion of the authors about the Curle formula not giving the correct result for the first example is incorrect. Their conclusion about the failure of the Curle formula for the second example is correct although it is based on their erroneous result when they use the Curle formula.

\subsection{Example 1- Scattering of a Plane Wave by a Rigid Sphere}

In this example, a plane wave with the wave number vector $\boldsymbol{k}=k \mathbf{e}_{1}$ is incident on a rigid sphere of radius $R_{0}$ with the center at the origin of the $\boldsymbol{x}$ frame. The unit vector along the $x_{1}$-axis is denoted as $\boldsymbol{e}_{1}$. The incident wave is approaching the sphere from the direction of negative $x_{1}$-axis with analytic expression

$$
p^{\prime}{ }_{i n c}(x, t)=\rho_{0} c_{0} U_{0} e^{i\left(k x_{1}-\omega t\right)}=P_{i n c} e^{-i \omega t}
$$

where $U_{0}$ is the velocity amplitude and $P_{i n c}$ is the complex amplitude of the incident wave. Before we get down to the algebraic manipulations, we discuss a subtle point about the application of the Curle formula here. Because we are working with linear acoustics, we replace $c_{0}^{2} \rho^{\prime}$ everywhere by the acoustic pressure $p^{\prime}$.

The Curle formula is valid for volume sources in the finite region and it is assumed that only outgoing waves exist in the problem. However, a plane wave approaching the sphere is radiated from a source at infinity and thus, strictly speaking, the Curle formula should not be used for this example. There is a way to handle this problem rigorously as follows. We assume that there is a monopole source on the negative $x_{1}$-axis very far from the origin of such an strength that near the origin the acoustic pressure from this source closely approximates the incidence wave of eq. (6). The governing wave equation which results in Curle's formula for this situation is:

$$
\square^{2} p^{\prime}{ }_{t o t}=Q \delta\left(x_{1}-x_{1 s}\right)-\frac{\partial}{\partial x_{i}}\left(p_{t o t}^{\prime} n_{i}^{\prime} \delta(f)\right)
$$


where $Q=Q(\boldsymbol{x}, t)$ is the monopole source strength located at $x_{1 s}$ on the negative $x_{1}$-axis very far from the origin. We note that $P_{i n c}$ is the solution of the following wave equation by assumption:

$$
\square^{2} p^{\prime}{ }_{\text {inc }}=Q \delta\left(x_{1}-x_{1 s}\right)
$$

Now using the relation $p^{\prime}{ }_{\text {tot }}=p_{\text {inc }}^{\prime}+p_{s c}^{\prime}$ in eq. (7) and then utilizing the relation of eq. (8), we get the following important result:

$$
\square^{2} p_{s c}^{\prime}=-\frac{\partial}{\partial x_{i}}\left(p_{t o t}^{\prime} n_{i}^{\prime} \delta(f)\right)
$$

Therefore, the Curle formula for this example is the solution of the above equation:

$$
4 \pi p_{s c}^{\prime}(\vec{x}, t)=\int_{S}\left(\frac{\left[p_{t o t}^{\prime}\right]_{r e t}}{r^{2}}+\frac{\left[\partial p_{t o t}^{\prime} / \partial \tau\right]}{r}\right) \cos \alpha d S
$$

where $\tau$ is the source time and $\alpha$ is the angle between $\boldsymbol{n}^{\prime}$, the outward unit normal to $S$, and the radiation direction $\boldsymbol{x}-\boldsymbol{y}$. In the far field and in terms of complex amplitudes, the Curle's formula can be written as follows from eq. (10):

$$
4 \pi P_{s c}=i k \int_{S} P_{t o t} \cos \alpha \frac{e^{i k|\boldsymbol{x}-\boldsymbol{y}|}}{|\boldsymbol{x}-\boldsymbol{y}|} d S
$$

We now introduce spherical polar coordinates with the polar axis as the positive $x_{1}$-axis. We avoid subscripted variables of Zinoviev and Bies by defining the spherical polar variables of the observer variable by $\boldsymbol{x}=(x, \Phi, \Theta)$ and source variable by $\boldsymbol{y}=\left(R_{0}, \phi, \theta\right)$.

With the assumption of $k R_{0} \ll 1$, we have [4]: 


$$
P_{\text {tot }}\left(R_{0}, \theta\right)=\rho_{0} c_{0} U_{0}\left(1+\frac{3}{2} i k R_{0} \cos \theta\right)
$$

The cosine of the angle between $\boldsymbol{n}^{\prime}$ and the radiation direction is given by

$$
\cos \alpha=\cos \theta \cos \Theta+\sin \theta \sin \Theta \cos (\Phi-\phi)
$$

and the element of the surface area of the sphere is given by

$$
d S=R_{0}^{2} \sin \theta d \theta d \phi
$$

We now approximate the radiation distance using the cosine rule for a triangle assuming that $x \gg R_{0}$. This gives us

$$
\begin{gathered}
|\boldsymbol{x}-\boldsymbol{y}| \approx x-R_{0} \cos \alpha \\
\frac{e^{i k|\boldsymbol{x}-\boldsymbol{y}|}}{|\boldsymbol{x}-\boldsymbol{y}|} \approx \frac{e^{i k x}}{x}\left(1-i k R_{0} \cos \alpha\right)
\end{gathered}
$$

Using eqs. (12) to (16) in the right side of the Curle formula, eq. (11), we must integrate the following fairly complicated integral:

$$
A \frac{e^{i k x}}{x} \int_{0}^{\pi} \int_{0}^{2 \pi}\left(1+\frac{3}{2} i k R_{0} \cos \theta\right)\left(1-i k R_{0} \cos \alpha\right) \cos \alpha \sin \theta d \theta d \phi \equiv I
$$

where the constant $A=i k \rho_{0} c_{0} U_{0} R_{0}^{2}$. The above integral can be easily integrated by Mathematica 5.0 and the result is:

$$
I=-\frac{4 \pi}{3} i k R_{0} A \frac{e^{i k x}}{x}\left(1-\frac{3}{2} \cos \Theta\right)
$$

Substituting for $A$ from above in this equation, the Curle formula, eq. (11) gives 


$$
P_{s c}=-\frac{1}{3} \rho_{0} c_{0} k^{2} U_{0} R_{0}^{3} \frac{e^{i k x}}{x}\left(1-\frac{3}{2} \cos \Theta\right)
$$

This is the correct result for the scattered pressure (see [4], page 427, eq. (91.8). Therefore, the Curle formula gives the correct result for this example.

In reading the paper of Zinoviev and Bies one does not see a result equivalent to our eq. (11) and the discussion leading to this result. Furthermore, they do not take the divergence of the integral on the right side of their eq. (37) analytically which is a trivial matter. Finally, they have made several errors in the evaluation of the integral based on the Curle formula.

\subsection{Example 2- Sound Generation by a Rigid Sphere in a Variable velocity Field}

The origin of this problem as discussed by the authors is somewhat confusing. Studying the surface pressure data used by the authors, we find that this example is on the determination of the radiation field of a rigid sphere oscillating with the velocity amplitude $U_{0}$ along the $x$-axis (see [6], page 284, Problem 1). In fact, the surface of the sphere can not be stationary because the momentum equation dictates that $\partial p^{\prime} / \partial n^{\prime}=0$ which is not what the authors assume (see eq. (57), Zinoviev and Bies). In this case, as we have shown above the Curle formula is not applicable but either the authors' eq. (4) or the solution of the linearized FW-H equation, our eq. (5), gives the correct result. We mention that the solution of the linearized FW-H equation is in fact the Kirchhoff formula [7]. We have integrated the integrals of the radiation formula for this example using Mathematica 5.0 proving the correctness of our assertion in agreement with the authors. We will not present the results here. As mentioned earlier, the evaluation of the integrals of the Curle formula for this example is also in error in the paper.

\subsection{Other Comments on This Paper}

1- The manipulations for converting the derivatives with respect to the source variable of the Lighthill stress tensor to derivatives with respect to the observer variable are not necessary. If $\psi_{i j}$ is the solution of the wave equation 


$$
\square^{2} \psi_{i j}=T_{i j}
$$

Then, taking $\partial^{2} / \partial x_{i} \partial x_{j}$ of both sides of this equation, we find that $\partial^{2} \psi_{i j} / \partial x_{i} \partial x_{j}$ is the solution of

$$
\square^{2} p^{\prime}=\frac{\partial^{2} T_{i j}}{\partial x_{i} \partial x_{j}}
$$

Since $\psi_{i j}$ is given as an integral by the solution of eq. (20), then the partial derivatives with respect to the observer variables in $\partial^{2} \psi_{i j} / \partial x_{i} \partial x_{j}$ will be outside the integral sign.

2. Most of the algebraic manipulations involving integration by parts in the paper are confusing and not mathematically precise because the authors do not clearly indicate evaluation of the integrand at the retarded time. For example, in the integrand of the integral on the left of eq. (5) of Zinoviev and Bies, the correct interpretation of the term involving the Lighthill stress tensor is $\left[\partial^{2} T_{i j} / \partial y_{i} \partial y_{j}\right]_{r e t}$. This means that in the algebraic manipulations leading to the right side of eq. (5), one must take account of the retarded time variable as follows

$\frac{1}{r}\left[\frac{\partial^{2} T_{i j}}{\partial y_{i} \partial y_{j}}\right]_{r e t}=\frac{\partial}{\partial y_{i}}\left(\left[\frac{\partial T_{i j}}{\partial y_{j}}\right]_{r e t} \frac{1}{r}\right)-\left(\frac{1}{c_{0} r}\left[\frac{\partial^{2} T_{i j}}{\partial \tau \partial y_{j}}\right]_{r e t}+\frac{1}{r}\left[\frac{\partial T_{i j}}{\partial y_{j}}\right]_{r e t}\right) \frac{\partial r}{\partial y_{i}}$

When the correct manipulations are performed, it can be shown that the right side of eq. (5) can be obtained with the retarded time correctly indicated. We have left out several steps here to get eq. (5) but they should be given to assure correctness of the final result. This remark applies also to other results involving integration by parts in the paper. The method selected by the authors for manipulating the integrals is complicated and prone to errors. A better approach will be working directly with the source terms of the wave 
equation, e.g., as in deriving the solution of our eq. (21), or working with the formal solution of the wave equation involving the Green's function before integrating the two delta functions in the integrand.

3. In the Subsection 3.3 Formulation of the divergence theorem, it is not at all clear what the authors mean by the discontinuity of the functions $F_{i}$ on the boundary $S_{v}$. The proper mathematical tool to study this problem is generalized functions $[8,9]$. The author of this letter has studied this problem in detail [10] and is unable to comprehend what the authors are implying in the paper. Also in differential geometry, the surface divergence has a clearly defined meaning [11, page 187]. The authors' definition does not correspond to this definition.

4. We are dismayed that the authors have neglected to do a detailed literature review of aeroacoustics before presenting their work to the public. The literature of acoustics on the subject of this paper is very extensive. The current state of the theoretical aeroacoustics is considerably more advanced than what is presented by these authors [12].

In summary, we have shown that the authors' improvement of the Curle formula is nothing more than the linear Kirchhoff formula for the density perturbations, added to the free-field solution of the nonlinear Lighthill's equation. As a general statement the authors' result is incorrect, although it reduces to the solution of the Ffowcs Williams-hawkings equation in the restricted context of linear acoustics. The derivation of the Curle formula for fixed surfaces [2] and the FW-H equation for moving surfaces [3], in contrast, are quite general and rest on solid physical and mathematical foundation.

\section{Acknowledgements}

The author has benefited greatly from the discussions with Professor Chris Morfey and Professor Geoffrey Lilley on the subject of this letter. The comments of Professor Morfey were particularly valuable to the author in writing this letter. The author thanks both these scientists for their invaluable help.

\section{References}

1. A. Zinoviev, D. A. Bies, On acoustic radiation by a rigid object in a fluid flow, Journal of Sound and Vibration 269 (2004) 535-548 
2. N. Curle, The influence of solid boundaries upon aerodynamic sound, Proceedings of the Royal Society of London A231 (1955) 505-514

3. J. E. Ffowcs Williams, D. L. Hawkings, Sound generation by turbulence and surfaces in arbitrary motion, Philosophical Transactions of the Royal Society of London A264 (1969) 321-514

4. A. D. Pierce: Acoustics: An Introduction to Its Physical Principles and Applications, Acoustical Society of America, New York, 1989

5. F. Farassat: The Kirchhoff Formulas For Moving Surfaces in Aeroacoustics - The Subsonic and Supersonic Cases, NASA Technical Memorandum 110285, 1996, Available at http://techreports.larc.nasa.gov/ltrs/PDF/NASA96-tm110285.pdf

6. L. D. Landau, E. M. Lifshitz: Fluid Mechanics, Course of Theoretical Physics, Vol. 6, Pergamon Press, Oxford, 1959

7. Kenneth S. Brentner, F. Farassat: An Analytical Comparison of the Acoustic Analogy and Kirchhoff Formulation for Moving Surfaces, AIAA Journal 36 (1998) 1379-1386, Available at http://techreports.larc.nasa.gov/ltrs/PDF/ 1998/jp/NASA-98-aj-ksb.pdf

8. I. M. Gel'fand, G. E. Shilov: Generalized Functions, Vol. 1- Properties and Operations, Academic Press, 1964

9. F. Farassat: Introduction to Generalized Functions With Applications in Aerodynamics and Aeroacoustics, Corrected Copy (April 1996), NASA Technical Paper 3428, 1994, Available at http://techreports.larc.nasa.gov/ltrs/ PDF/tp3428.pdf

10 F. Farassat, M. K. Myers: An Analysis of the Quadrupole Noise Source of High Speed Rotating Blades, in Computational Acoustics - Scattering, Gaussian Beams, and Aeroacoustics, D. Lee, A. Cakmak, R Vichnevetsky (editors), Vol. 2 (1990) 227-240

11. A. J. McConnell: Applications of Tensor Analysis, Dover Books, New York, 1957 
12. Harvey H. Hubbard (editor): Aeroacoustics of Flight Vehicles- Theory and Practice, 2 Volumes, Acoustical Society of America, 1995 